\title{
Magnetoelectric effects in gyrotropic superconductors
}

\author{
Wen-Yu He $\odot$ and K. T. Law ${ }^{*}$ \\ Department of Physics, Hong Kong University of Science and Technology, Clear Water Bay, Hong Kong, China
}

(Received 16 February 2019; revised manuscript received 28 February 2020; accepted 4 March 2020; published 20 March 2020)

\begin{abstract}
The magnetoelectric effect (or Edelstein effect) in noncentrosymmetric superconductors states that a supercurrent can induce spin magnetization. This is an intriguing phenomenon which has potential applications in superconducting spintronic devices. However, the original Edelstein effect only applies to superconductors with polar point group symmetry. In recent years, many new noncentrosymmetric superconductors have been discovered, such as superconductors with chiral lattice symmetry and superconducting transition metal dichalcogenides with various lattice structures. In this Rapid Communication, we provide a general framework to describe the supercurrent-induced magnetization in these recently discovered superconductors with gyrotropic point groups.
\end{abstract}

DOI: 10.1103/PhysRevResearch.2.012073

\section{INTRODUCTION}

In noncentrosymmetric metals, the spin and momentum of electrons are coupled such that the deformation of the Fermi surface due to dissipative current can polarize electron spins, and this is called the magnetoelectric effect or the Edelstein effect [1-3]. More interestingly, in some noncentrosymmetric superconductors, supercurrents can also give rise to a static magnetization without dissipation [3-8]. Conversely, a static magnetization can drive local supercurrents [7,9-13]. As pointed out by Edelstein [5,6], in superconductors with a polar axis $c$ and Rashba spin-orbit coupling (SOC), the magnetization $\boldsymbol{M}$ induced by supercurrents can be expressed as $\boldsymbol{M} \propto \boldsymbol{c} \times \boldsymbol{J}^{\mathrm{S}}$ [5], where $\boldsymbol{J}^{\mathrm{S}}$ is the supercurrent density. In such Rashba superconductors, the induced magnetization is always perpendicular to the direction of the supercurrent and the polar axis, as is shown in Fig. 1(a).

In recent years, many noncentrosymmetric superconductors such as superconductors with chiral lattice structures [14-17], and superconducting transition metal dichalcogenides (TMDs) [18-24] have been discovered. Since these emergent noncentrosymmetric superconductors have different crystal symmetries, the SOC in these materials has different forms. For example, superconductors with point group symmetry $T$ and $O$, which belong to chiral (or enantiomorphic) point groups, have isotropic SOC in the form of $v \boldsymbol{p} \cdot \boldsymbol{\sigma}$, where $\boldsymbol{p}$ is momentum, $\boldsymbol{\sigma}$ is the Pauli matrices, and $v$ is the SOC strength [25]. On the other hand, multilayer $1 T_{d^{-}}$

\footnotetext{
*Author to whom correspondence should be addressed: phlaw@ust.hk

Published by the American Physical Society under the terms of the Creative Commons Attribution 4.0 International license. Further distribution of this work must maintain attribution to the author(s) and the published article's title, journal citation, and DOI.
}

structure $\mathrm{WTe}_{2}$ and $\mathrm{MoTe}_{2}$ possess anisotropic SOC [24]. For atomically thin monolayer $2 \mathrm{H}-\mathrm{MoS}_{2}$ and $2 \mathrm{H}-\mathrm{NbSe}_{2}$, Ising SOC [18-20,26-28] is present which pins electron spins to the out-of-plane directions. These different forms of SOC are expected to cause unconventional magnetoelectric effects different from the one in Fig. 1(a) for Rashba superconductors. However, a general understanding of the magnetoelectric effects of all these noncentrosymmetric superconductors is lacking. In this Rapid Communication, through linear response theory and group theory analysis, we provide a general and powerful way to understand magnetoelectric effects in noncentrosymmetric superconductors. Importantly, we point out that in superconducting chiral crystals and superconducting quasi-two-dimensional (2D) TMDs, applying a supercurrent can give the unconventional magnetization shown in Figs. 1(b) and 1 (c), respectively.

First of all, we note that among the superconductors within the 21 noncentrosymmetric point groups, the magnetoelectric effect is generally nonzero only for the ones belonging to the 18 gyrotropic point groups [29,30]-we call these superconductors gyrotropic superconductors. The explicit forms of the magnetoelectric pseudotensors for the 18 gyrotropic point groups are listed in Table I. According to Table I, many of the magnetoelectric responses of noncentrosymmetric superconductors can be identified immediately.

For example, for materials belonging to the $T$ and $O$ point groups, such as $\mathrm{Li}_{2} \mathrm{Pd}_{3} \mathrm{~B}, \mathrm{Li}_{2} \mathrm{Pt}_{3} \mathrm{~B}[14,15]$, and $\mathrm{Mo}_{3} \mathrm{Al}_{2} \mathrm{C}$ [16], the magnetoelectric effect is purely longitudinal, meaning that the induced spin magnetization is always parallel to the direction of the supercurrent, as is shown in Fig. 1(b). This is a quantum analog of classical solenoids in which the induced magnetic field is parallel to the current directions. We further point out that, for quasi-two-dimensional materials, the only symmetries which allow one to have induced spin magnetization perpendicular to the atomic plane are the ones which have the polar axis lying inside the atomic plane. The recently discovered multilayer $1 T_{d}$-structure $\mathrm{WTe}_{2}[22,23]$ and $\mathrm{MoTe}_{2}$ [24] naturally fulfils this condition so that there 


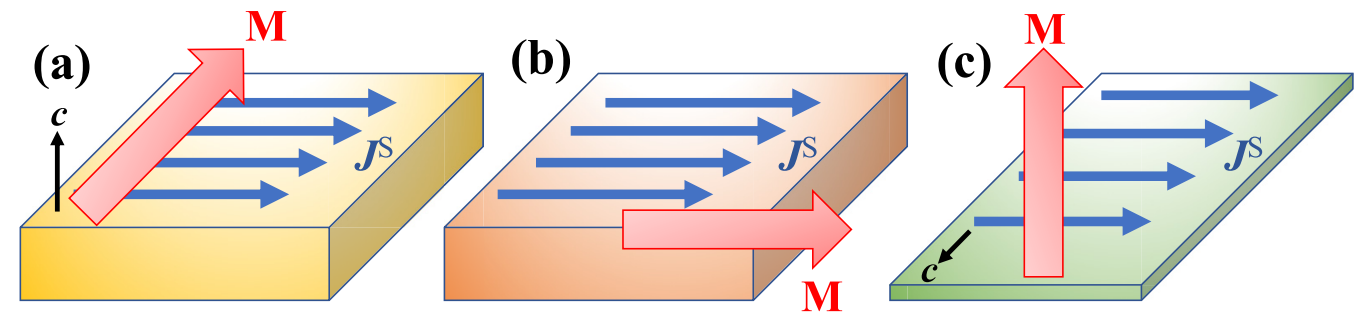

FIG. 1. The supercurrent driven magnetization in (a) polar superconductors with Rashba SOC, (b) superconducting chiral crystals belonging to $T$ and $O$ point groups, and (c) quasi-2D superconducting TMDs with the polar axis lying inside the atomic plane. In polar superconductors with Rashba SOC, the supercurrent driven magnetization $\boldsymbol{M}$ is the transverse response as $\boldsymbol{M} \propto \boldsymbol{c} \times \boldsymbol{J}^{\mathrm{S}}$. In superconducting chiral crystals of $T$ and $O$ point groups, the magnetization arising from the supercurrent is parallel to the supercurrent direction and the magnetoelectric effect becomes a longitudinal response. In quasi-2D superconducting TMDs with $C_{1}, C_{2}, C_{1 v}$, or $C_{2 v}$ point group symmetry, the polar axis $c$ lies inside the atomic plane and allows the in-plane supercurrent to induce the out-of-plane magnetization.

exists an in-plane supercurrent-induced out-of-plane magnetization seen from Fig. 1(c).

TABLE I. List of magnetoelectric pseudotensor $T$ for the noncentrosymmetric superconductors in the 18 gyrotropic point groups. $T_{i j}$ with $i, j=x, y, z$ are in general the elements in $T . T_{\|}$denotes the elements $T_{x x}, T_{y y}$ as $T_{\|}=T_{x x}= \pm T_{y y}$ and $T_{0}$ denotes the diagonal elements in the $T$ and $O$ point group when $T_{x x}=T_{y y}=T_{z z} . T_{\mathrm{d}}$ and $T^{-}$denote the symmetric and antisymmetric off-diagonal elements in $T$, respectively. The principal axis is along the $z$ direction.

\begin{tabular}{|c|c|c|c|c|c|c|c|}
\hline Point group & & $T_{i j}$ & & Point group & & $T_{i j}$ & \\
\hline$C_{1}$ & $\left(\begin{array}{l}T_{x x} \\
T_{y x} \\
T_{z x}\end{array}\right.$ & $\begin{array}{l}T_{x y} \\
T_{y y} \\
T_{z y}\end{array}$ & $\left.\begin{array}{l}T_{x z} \\
T_{y z} \\
T_{z z}\end{array}\right)$ & $C_{2}$ & $\left(\begin{array}{c}T_{x x} \\
T_{y x} \\
0\end{array}\right.$ & $\begin{array}{c}T_{x y} \\
T_{y y} \\
0\end{array}$ & $\left.\begin{array}{c}0 \\
0 \\
T_{z z}\end{array}\right)$ \\
\hline$C_{3}$ & $\left(\begin{array}{c}T_{\|} \\
T^{-} \\
0\end{array}\right.$ & $\begin{array}{c}-T^{-} \\
T_{\|} \\
0\end{array}$ & $\left.\begin{array}{c}0 \\
0 \\
T_{z z}\end{array}\right)$ & $C_{4}$ & $\left(\begin{array}{c}T_{\|} \\
T^{-} \\
0\end{array}\right.$ & $\begin{array}{c}-T^{-} \\
T_{\|} \\
0\end{array}$ & $\left.\begin{array}{c}0 \\
0 \\
T_{z z}\end{array}\right)$ \\
\hline$C_{6}$ & $\begin{array}{c}T_{\|} \\
T^{-} \\
0\end{array}$ & $\begin{array}{c}-T^{-} \\
T_{\|} \\
0\end{array}$ & $\left.\begin{array}{c}0 \\
0 \\
T_{z z}\end{array}\right)$ & $C_{1 v}$ & $\left(\begin{array}{c}0 \\
T_{y x} \\
0\end{array}\right.$ & $\begin{array}{c}T_{x y} \\
0 \\
T_{z y}\end{array}$ & $\left.\begin{array}{c}0 \\
T_{y z} \\
0\end{array}\right)$ \\
\hline$C_{2 v}$ & $\left(\begin{array}{c}0 \\
T_{y x} \\
0\end{array}\right.$ & $\begin{array}{c}T_{x y} \\
0 \\
0\end{array}$ & $\left.\begin{array}{l}0 \\
0 \\
0\end{array}\right)$ & $C_{3 v}$ & $\left(\begin{array}{c}0 \\
T^{-} \\
0\end{array}\right.$ & $\begin{array}{c}-T^{-} \\
0 \\
0\end{array}$ & $\left.\begin{array}{l}0 \\
0 \\
0\end{array}\right)$ \\
\hline$C_{4 v}$ & $\left(\begin{array}{c}0 \\
T^{-} \\
0\end{array}\right.$ & $\begin{array}{r}-T^{-} \\
0 \\
0\end{array}$ & $\left.\begin{array}{l}0 \\
0 \\
0\end{array}\right)$ & $C_{6 v}$ & $\left(\begin{array}{c}0 \\
T^{-} \\
0\end{array}\right.$ & $\begin{array}{c}-T^{-} \\
0 \\
0\end{array}$ & $\left.\begin{array}{l}0 \\
0 \\
0\end{array}\right)$ \\
\hline$D_{2 d}$ & $\left(\begin{array}{c}T_{\|} \\
0 \\
0\end{array}\right.$ & $\begin{array}{c}0 \\
-T_{\|} \\
0\end{array}$ & $\left.\begin{array}{l}0 \\
0 \\
0\end{array}\right)$ & $S_{4}$ & $\left(\begin{array}{c}T_{\|} \\
T_{\mathrm{d}} \\
0\end{array}\right.$ & $\begin{array}{c}T_{\mathrm{d}} \\
-T_{\|} \\
0\end{array}$ & $\left.\begin{array}{l}0 \\
0 \\
0\end{array}\right)$ \\
\hline$D_{2}$ & $\left(\begin{array}{c}T_{x x} \\
0 \\
0\end{array}\right.$ & $\begin{array}{c}0 \\
T_{y y} \\
0\end{array}$ & $\left.\begin{array}{c}0 \\
0 \\
T_{z z}\end{array}\right)$ & $D_{3}$ & $\left(\begin{array}{c}T_{\|} \\
0 \\
0\end{array}\right.$ & $\begin{array}{c}0 \\
T_{\|} \\
0\end{array}$ & $\left.\begin{array}{c}0 \\
0 \\
T_{z z}\end{array}\right)$ \\
\hline$D_{4}$ & $\left(\begin{array}{c}T_{\|} \\
0 \\
0\end{array}\right.$ & $\begin{array}{c}0 \\
T_{\|} \\
0\end{array}$ & $\left.\begin{array}{c}0 \\
0 \\
T_{z z}\end{array}\right)$ & $D_{6}$ & $\left(\begin{array}{c}T_{\|} \\
0 \\
0\end{array}\right.$ & $\begin{array}{c}0 \\
T_{\|} \\
0\end{array}$ & $\left.\begin{array}{c}0 \\
0 \\
T_{z z}\end{array}\right)$ \\
\hline$T$ & $\left(\begin{array}{c}T_{0} \\
0 \\
0\end{array}\right.$ & $\begin{array}{c}0 \\
T_{0} \\
0\end{array}$ & $\left.\begin{array}{c}0 \\
0 \\
T_{0}\end{array}\right)$ & $O$ & $\left(\begin{array}{c}T_{0} \\
0 \\
0\end{array}\right.$ & $\begin{array}{c}0 \\
T_{0} \\
0\end{array}$ & $\left.\begin{array}{c}0 \\
0 \\
T_{0}\end{array}\right)$ \\
\hline
\end{tabular}

On the other hand, for Ising superconductors such as $2 \mathrm{H}$-structure $\mathrm{MoS}_{2}$ and $\mathrm{NbSe}_{2}$, the magnetoelectric effect is indeed zero due to their $D_{3 h}$ point group, even though the SOC in these materials is particularly strong. These $2 H$-TMDs are interesting examples of noncentrosymmetric superconductors which give rise to a zero magnetoelectric response. Importantly, under uniaxial strain which reduces $D_{3 h}$ to $C_{2 v}$, a purely out-of-plane magnetization can be induced by a supercurrent in $2 H$-TMD. Therefore, our results, as summarized in Table I, can be used to provide the guiding principles to generate and manipulate magnetoelectric responses in superconductors.

The rest of this Rapid Communication is organized as follows. First, we construct the microscopic model for the magnetoelectric effect and show that the induced spin magnetization is related to the supercurrent by a rank-two pseudotensor $T_{i j}$. Second, we analyze the symmetry properties of $T_{i j}$ under different point group operations and list the general forms of $T_{i j}$ in Table I. Third, we discuss the application of Table I in the understanding of several interesting superconductors with a gyrotropic point group, including a superconductor with chiral lattice symmetry and superconducting TMDs. Finally, we demonstrate how an unconventional magnetoelectric response can be generated by strain in nongyrotropic superconductors using the $2 H$-structure TMD as an example.

\section{RESULTS}

\section{A. Theory for the superconducting magnetoelectric effect}

To study a noncentrosymmetric superconductor which carries a supercurrent, we consider the Bogliubov-de Gennes Hamiltonian

$$
H(\boldsymbol{p}, \boldsymbol{q})=\left(\begin{array}{cc}
\xi_{\boldsymbol{p}+\frac{1}{2} \boldsymbol{q}}+\boldsymbol{g}_{\boldsymbol{p}+\frac{1}{2} \boldsymbol{q}} \cdot \boldsymbol{\sigma} & i \Delta_{\boldsymbol{q}} \sigma_{y} \\
-i \Delta_{\boldsymbol{q}}^{*} \sigma_{y} & -\xi_{-\boldsymbol{p}+\frac{1}{2} \boldsymbol{q}}-\boldsymbol{g}_{-\boldsymbol{p}+\frac{1}{2} \boldsymbol{q}} \cdot \boldsymbol{\sigma}^{*}
\end{array}\right) .
$$

Here, the Nambu basis is $\left[c_{\boldsymbol{p}+\frac{1}{2} \boldsymbol{q}, \uparrow}^{\dagger}, c_{\boldsymbol{p}+\frac{1}{2} \boldsymbol{q}, \downarrow}^{\dagger}, c_{-\boldsymbol{p}+\frac{1}{2} \boldsymbol{q}, \uparrow}, c_{-\boldsymbol{p}+\frac{1}{2} \boldsymbol{q}, \downarrow}\right]$, with $c_{\boldsymbol{p}^{\prime}, s}^{(\dagger)}$ the annihilation (creation) operator at the momentum $\boldsymbol{p}^{\prime}$ and spin index $s=\uparrow / \downarrow$. The kinetic energy of an electron with momentum $\boldsymbol{p}$ is described by $\xi_{p}$. The vector $\boldsymbol{g}_{p}$ describes the SOC of the material and its specific form is determined by the lattice symmetry, and $\sigma$ denotes the Pauli matrices. From the definition of the basis, it is clear that the $\Delta_{q} \sigma_{y}$ term pairs 
electrons with net momentum $\boldsymbol{q}$ and opposite spin. Therefore, the Hamiltonian describes a superconductor which sustains a supercurrent when $\boldsymbol{q}$ is finite. With the Hamiltonian, we can calculate the spin magnetization $\boldsymbol{M}$ as [31]

$$
M_{k}=-\left.\frac{1}{2 \beta V} \sum_{\boldsymbol{p}, \boldsymbol{q}, n} \frac{\partial}{\partial h_{k}} \operatorname{tr} \log G^{-1}\left(\boldsymbol{p}, \boldsymbol{q}, i \omega_{n}\right)\right|_{\boldsymbol{h}=\mathbf{0}} .
$$

Here, $\beta$ reads $\beta^{-1}=k_{b} T$, with $k_{b}$ the Boltzmann constant, $k=x, y, z$ denotes the component of $\boldsymbol{M}$, and the Matsubara Green's function is defined as

$$
G^{-1}\left(\boldsymbol{p}, \boldsymbol{q}, i \omega_{n}\right)=i \omega_{n}-H(\boldsymbol{p}, \boldsymbol{q})-\frac{1}{2} g \mu_{\mathrm{B}}\left(\begin{array}{cc}
\boldsymbol{h} \cdot \boldsymbol{\sigma} & 0 \\
0 & -\boldsymbol{h} \cdot \boldsymbol{\sigma}^{*}
\end{array}\right),
$$

with $\boldsymbol{h}$ describing a Zeeman field, $\mu_{\mathrm{B}}$ the Bohr magneton, and $g$ the Landé $g$ factor. Since the supercurrent associated finite momentum $\boldsymbol{q}$ couples with the velocity operator as $\nabla\left(\xi_{p}+g_{p} \cdot \sigma\right) \cdot \frac{1}{2} q$ at the linear order, the spin magnetic moment is induced through the rank-two pseudotensor $J_{i j}(\boldsymbol{p})=$ $\partial_{p_{j}} g_{i}(\boldsymbol{p})$, with $i, j=x, y, z$. As a result, in the presence of a supercurrent, the coupling term $\sigma_{i} J_{i j} \frac{1}{2} q_{j}$ acts as an effective Zeeman field that generates the spin magnetization.

In order to obtain an analytical form for the supercurrentinduced magnetization $\boldsymbol{M}$, we expand $\operatorname{tr} \log G^{-1}\left(\boldsymbol{p}, \boldsymbol{q}, i \omega_{n}\right)$ in terms of the Zeeman field $\boldsymbol{h}$ and the pairing order parameters $\Delta_{q}$ and $\Delta_{q}^{*}$. Then, by taking the derivative on the Zeeman field $\boldsymbol{h}$ at $\boldsymbol{h}=\boldsymbol{\mathbf { 0 }}$ and summing over $\boldsymbol{p}, \boldsymbol{q}$, the Matsubara frequencies $\omega_{n}$, we obtain the analytical form for the magnetization $M$, which can be written as $M_{k}=T_{k j} \tilde{J}_{j}^{\mathrm{S}}$, where $J^{\mathrm{S}}$ is the supercurrent density. We denote $T_{k j}$ as a component of the magnetoelectric pseudotensor $T$ [32] with the form

$$
\begin{aligned}
T_{k j}= & \left\{\frac{\Delta_{0}^{2}}{\boldsymbol{g}_{\boldsymbol{p}_{\mathrm{F}}}^{2}} f\left(\frac{\left|\boldsymbol{g}_{\boldsymbol{p}_{\mathrm{F}}}\right|}{\pi k_{b} T_{c}}\right)\left\langle J_{k j}\left(\boldsymbol{p}_{\mathrm{F}}\right)\right\rangle+\left[\frac{7 \Delta_{0}^{2} \zeta(3)}{8 \pi^{2} k_{b}^{2} T_{c}^{2}}\right.\right. \\
& \left.-\frac{\Delta_{0}^{2}}{\boldsymbol{g}_{\boldsymbol{p}_{\mathrm{F}}}^{2}} f\left(\frac{\left|\boldsymbol{g}_{\boldsymbol{p}_{\mathrm{F}}}\right|}{\pi k_{b} T_{c}}\right)\right]\left\langle\hat{\boldsymbol{g}}_{\boldsymbol{p}_{\mathrm{F}} k} \hat{\boldsymbol{g}}_{\boldsymbol{p}_{\mathrm{F}} i} J_{i j}\left(\boldsymbol{p}_{\mathrm{F}}\right)\right\rangle \frac{2 \mu_{\mathrm{B}} g}{3 \sqrt{3} \xi} N\left(E_{\mathrm{F}}\right) .
\end{aligned}
$$

Here, $\boldsymbol{p}_{\mathrm{F}}$ represents the Fermi momentum, $N\left(E_{\mathrm{F}}\right)$ is the density of states at the Fermi level, $\Delta_{0}$ is the homogeneous pairing order parameter magnitude, $\xi$ is the superconducting coherence length, and $\tilde{\boldsymbol{J}}^{\mathrm{S}}=\boldsymbol{J}^{\mathrm{S}} / J_{\max }^{\mathrm{S}}$ is the supercurrent density normalized by the maximum supercurrent density $J_{\max }^{\mathrm{S}}=$ $2 e \Delta_{0}^{2} \frac{2 \hbar}{3 \sqrt{3} m^{*} \xi}$, with $m^{*}$ being the effective pairing mass [33]. We have \langle\rangle$=\int \frac{d \Omega_{p_{\mathrm{F}}}}{4 \pi}$ as the angle average at the Fermi surface and define the function $f(\rho)$ as

$$
f(\rho)=\operatorname{Re} \sum_{n=0}^{\infty}\left(\frac{1}{2 n+1}-\frac{1}{2 n+1+i \rho}\right) .
$$

The magnetoelectric susceptibility obtained in Eq. (4) applies for superconductors with a pair of spin splitted bands. For a noncentrosymmetric superconductor with multiple bands, the total magnetoelectric susceptibility is the sum of the contribution from all the paired Fermi pockets, as is discussed in the Supplemental Material [32]. As the magnetoelectric pseudotensor $T$ is constructed from the SOC pseudovector $\boldsymbol{g}_{\boldsymbol{p}_{\mathrm{F}}}$ at the Fermi surface, its general form is determined by the crystal point group symmetry, as shown in the next section.

\section{B. Symmetry analysis for magnetoelectric pseudotensor in three dimensions}

The SOC pseudovector $\boldsymbol{g}_{\boldsymbol{p}}$ under the point group operation $\hat{R}$ respects $\boldsymbol{g}(\boldsymbol{p})=\operatorname{det}(\hat{R}) \hat{R} \boldsymbol{g}\left(\hat{R}^{-1} \boldsymbol{p}\right)[34,35]$. Hence inheriting from the pseudovector $g_{p}$, we show that the magnetoelectric pseudotensor $T_{k j}$ under the crystal symmetry is subject to the constraints [32]

$$
T=\operatorname{det}(\hat{R}) \hat{R} T \hat{R}^{\mathrm{T}}
$$

where $\hat{R}$ is the orthogonal matrix of the point group transformation. The general derivation of Eq. (6) for all the noncentrosymmetric point groups is given in the Materials and Methods section. All the nonzero components of the magnetoelectric pseudotensor $T$ of the 18 gyrotropic point groups are listed in Table I.

\section{Unconventional magnetoelectric effects in gyrotropic superconductors}

With the general form of $T_{i j}$ in Table I, some unconventional magnetoelectric responses of gyrotropic superconductors can be identified immediately. One particular interesting case is for materials with point group symmetry $T$ and $O$. In this case, the tensor $T_{i j}$ is proportional to the identity matrix and implies that the induced magnetization is parallel to the supercurrent direction shown in Fig. 1(b), namely, $\boldsymbol{M} \propto \tilde{\boldsymbol{J}}^{\mathrm{S}}$. This is a quantum analog of classical solenoids but without the need to fabricate any helical structures, and the longitudinal response is induced by SOC. It is interesting to note that a few superconductors with relatively high $T_{c}$ have point group $O$ such as $\mathrm{Li}_{2} \mathrm{Pt}_{3} \mathrm{~B}, \mathrm{Li}_{2} \mathrm{Pd}_{3} \mathrm{~B}$, and $\mathrm{Mo}_{3} \mathrm{Al}_{2} \mathrm{C}$ [14-16]. Other interesting examples are superconducting $\mathrm{TaRh}_{2} \mathrm{~B}_{2}$ and $\mathrm{NbRh}_{2} \mathrm{~B}_{2}$ which had been recently discovered by Carnicom et al. [17]. These superconductors have point group $C_{3}$. From Table I, we immediately realize that a supercurrent along the $z$ direction (the principal symmetry axis direction) will generate a pure longitudinal response such that the supercurrent-induced magnetization is parallel to the direction of the supercurrent. On the other hand, a supercurrent perpendicular to the $z$ direction generates a magnetization in the $x-y$ plane.

Importantly, in the normal state, these materials with chiral lattice symmetries are Kramers Weyl semimetals which have Weyl points pinned at time-reversal invariant momenta [25]. Therefore, using Table I, one can immediately identify the unconventional magnetoelectric properties of a superconducting Kramers Weyl semimetal. More noncentrosymmetric superconductors with gyrotropic point groups are listed in the Supplemental Material [32].

Another interesting result from Table $I$ is that, in quasi two dimensions, only $C_{1}, C_{1 v}, C_{2 v}$, and $C_{2}$ symmetries with the polar axis lying inside the atomic plane allow an out-ofplane magnetization induced by an in-plane supercurrent, as is present in Fig. 1(c). Interestingly, the recently discovered few-layer $1 T_{d}-\mathrm{WTe}_{2}[22,23]$ and $1 T_{d}-\mathrm{MoTe}_{2}$ [24] have such a low symmetry: $C_{1 v}$. Moreover, due to the large SOC in these materials, the magnetoelectric effect is expected to be strong. 


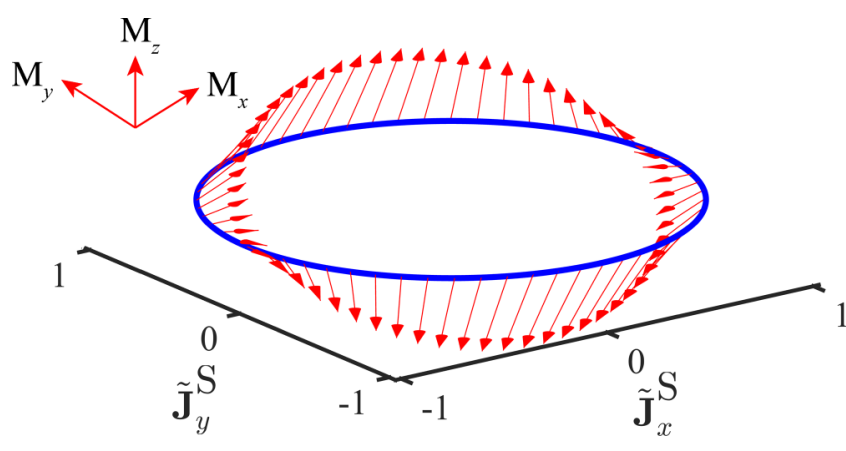

FIG. 2. The magnetization direction (red arrows) can be controlled by the in-plane supercurrent directions $\tilde{\boldsymbol{J}}^{\mathrm{S}}$ as calculated using realistic parameters for bilayer $1 T_{d}-\mathrm{MoTe}_{2}$. The in-plane mirror invariant line is defined along the $\tilde{\boldsymbol{J}}_{x}^{\mathrm{S}}$ direction.

With $C_{1 v}$ symmetry, we show below how the magnetization direction can also be controlled by the direction of the supercurrent. The magnetoelectric effects of $2 H$-structure TMDs are also discussed in the next section.

\section{Unconventional magnetoelectric effects of superconducting TMDs}

In this section, we apply the general theory obtained above to two interesting TMDs, namely, bilayer or multilayer $1 T_{d^{-}}$ $\mathrm{MoTe}_{2}$ and strained monolayer $2 \mathrm{H}-\mathrm{NbSe}_{2}$. For the case of bilayer $1 T_{d}-\mathrm{MoTe}_{2}$, the crystal structure belongs to the $C_{1 v}$ point group and only has one in-plane mirror symmetry with $y \rightarrow-y$. The superconductivity occurs at the critical temperature $T_{c}=4 \mathrm{~K}$ and it has the in-plane anisotropic upper critical field $H_{c 2}$ which exceeds the Pauli limit $H_{p}$ due to its anisotropic SOC $\boldsymbol{g}\left(p_{x}, p_{y}\right)=\left(v_{x} p_{y}, v_{y} p_{x}, v_{z} p_{y}\right)$. From the angle-dependent in-plane $H_{c 2}(\phi)$, the anisotropic SOC strength at the Fermi level is estimated to be $\boldsymbol{g}(\phi)=$ (16.9 $\sin \phi, 23.5 \cos \phi, 23.1 \sin \phi) \mathrm{meV}$ [24]. With the Fermi wave vector $\left(p_{\mathrm{F} x}, p_{\mathrm{F} y}\right)=(1.009,1.050) \mathrm{nm}^{-1}$ and the Fermi energy density of states $N\left(E_{\mathrm{F}}\right)=4.3776 \mathrm{eV}^{-1} \mathrm{~nm}^{-2}$ from the first-principles calculation [24], we can further use the pairing gap $\Delta_{0}=1.76 k_{b} T_{c}$ at zero temperature and the coherence length $\xi=20.79 \mathrm{~nm}$ [24] to estimate the magnetoelectric pseudotensor $T$ as $T_{x y}=2.52 \times 10^{-4} \mu_{\mathrm{B}} / \mathrm{nm}^{2}, T_{y x}=2.64 \times$ $10^{-4} \mu_{\mathrm{B}} / \mathrm{nm}^{2}$, and $T_{z y}=3.43 \times 10^{-3} \mu_{\mathrm{B}} / \mathrm{nm}^{2}$ while all other elements are zero.

From the form of $T$, we note that the supercurrent-induced magnetization $\left(M_{x}, M_{y}, M_{z}\right)$ is a function of the in-plane supercurrent direction shown in Fig. 2. When the supercurrent flows along the $y$ direction, the $z$-direction magnetization $M_{z}$ gets the optimized value. For a supercurrent close to the critical value, the maximum $M_{z}$ can reach $M_{z}=7.6 \times$ $10^{-4} \mu_{\mathrm{B}}$ per unit cell. The strength of the supercurrent-induced magnetization is in the same order as $\mathrm{Bi} / \mathrm{Ag}$ bilayers with strong Rashba SOC [36,37] which has been observed [38]. Therefore, we expect that $1 T_{d}-\mathrm{MoTe}_{2}$ is an excellent candidate to observe the superconducting magnetoelectric effect which has yet to be observed experimentally. Moreover, our prediction of the supercurrent-induced magnetization also applies to the recently realized superconducting $1 T_{d}-\mathrm{WTe}_{2}[22,23]$.

For the case of $2 \mathrm{H}-\mathrm{NbSe}_{2}$ which is also known as an Ising superconductor [18-20,27,28], the materials have point group symmetry $D_{3 h}$ [26]. Due to the in-plane mirror invariant line at $x=0$ and the threefold rotation symmetry, the SOC takes the form $g_{z}(\boldsymbol{p}) \propto p_{x}\left(p_{x}^{2}-3 p_{y}^{2}\right)$ around the $\Gamma$ pocket and $g_{z}(\boldsymbol{p} \mp K)= \pm \beta_{\text {so }}$ around the $K\left(K^{\prime}\right)$ pockets [28]. According to the general analysis above, the threefold rotation symmetry forces the magnetoelectric pseudotensor $T$ to be zero and there is no magnetoelectric effect. This is an interesting example of a noncentrosymmetric superconductor with zero magnetoelectric response. Interestingly, a uniaxial strain breaks the threefold symmetry and reduces the point group symmetry to $C_{2 v}$ with the polar axis lying inside the atomic plane. As a result, SOC which is linearly proportional to $p_{x}$, namely, $g_{z}(\boldsymbol{p}) \propto p_{x}$, can be induced. Therefore, under uniaxial strain, the magnetoelectric pseudotensor $T$ will have a nonzero element $T_{z x}$ and the $x$ component of the supercurrent will generate the out-of-plane magnetization. This provides another way to use strain to generate and manipulate spin polarizations in nongyrotropic superconductors.

\section{DISCUSSION}

In this Rapid Communication, we presented the general form of the magnetoelectric pseudotensor for gyrotropic superconductors as summarized in Table I. Our theory provides a powerful tool for the search for unconventional magnetoelectric effects (or the Edelstein effect) in materials. Guided by the general theory, we demonstrated different ways of generating and manipulating spin polarizations in noncentrosymmetric superconductors such as $1 T_{d}-\mathrm{MoTe}_{2}$ and $2 H$ $\mathrm{NbSe}_{2}$. In particular, we predict that $1 T_{d}-\mathrm{MoTe}_{2}$ is an excellent candidate for the realization of the Edelstein effect in superconductors.

Importantly, Table I can be used to identify the magnetoelectric effects of a large number of superconductors with chiral lattice symmetry such as $\mathrm{Li}_{2} \mathrm{Pt}_{3} \mathrm{~B}, \mathrm{Li}_{2} \mathrm{Pd}_{3} \mathrm{~B}$, and $\mathrm{Mo}_{3} \mathrm{Al}_{2} \mathrm{C}$ with $O$ point group [14-16], and the recently discovered superconducting chiral crystals such as $\mathrm{TaRh}_{2} \mathrm{~B}_{2}$ and $\mathrm{NbRh}_{2} \mathrm{~B}_{2}$ with $C_{3}$ point group [17]. The supercurrent driven longitudinal magnetization in those chiral crystals resembles the current-induced magnetization of solenoids at a microscopic scale. These superconducting chiral crystals can have potential applications for designing future superconducting spintronic devices [39].

It is also important to note that the Edelstein effect (currentinduced spin magnetization effect) had been observed in several materials with Rashba SOC in the normal state [40]. However, the Edelstein effect has not been observed in superconducting materials. Here, we identified several superconducting materials, such as $1 T_{d}-\mathrm{MoTe}_{2}$ and strained $\mathrm{NbSe}_{2}$, which possess a strong Edelstein effect and the proposed Edelstein effect can be observed through magneto-optical Kerr effect measurements [41].

\section{ACKNOWLEDGMENTS}

W.-Y.H. and K.T.L. are thankful for the support of HKRGC through C6026-16W, 16324216, 16307117, and 16309718. K.T.L. is further supported by the Croucher Foundation and the Dr. Tai-chin Lo Foundation. 
[1] L. S. Levitov, Yu. V. Nazarov, and G. M. Eliashberg, Zh. Eksp. Teor. Fiz. 88, 229 (1985) [Sov. Phys. JETP 61, 133 (1985)].

[2] V. M. Edelstein, Solid State Commun. 73, 233 (1990).

[3] M. Fiebig, J. Phys. D 38, R123 (2005).

[4] L. S. Levitov, Yu. V. Nazarov, and G. M. Eliashberg, Pis'ma Zh. Eksp. Teor. Fiz. 41, 365 (1985) [JETP Lett. 41, 445 (1985)].

[5] V. M. Edel'shtein, Zh. Eksp. Teor. Fiz. 95, 2151 (1989) [Sov. Phys. JETP 68, 1244 (1989)].

[6] V. M. Edelstein, Phys. Rev. Lett. 75, 2004 (1995).

[7] S. K. Yip, Phys. Rev. B 65, 144508 (2002).

[8] S. Fujimoto, Phys. Rev. B 72, 024515 (2005).

[9] K. V. Samokhin, Phys. Rev. B 70, 104521 (2004).

[10] R. P. Kaur, D. F. Agterberg, and M. Sigrist, Phys. Rev. Lett. 94, 137002 (2005).

[11] O. Dimitrova and M. V. Feigelman, Phys. Rev. B 76, 014522 (2007).

[12] E. Bauer and M. Sigrist, Noncentrosymmetric Superconductors (Springer, Berlin, 2012).

[13] S. Mironov and A. Buzdin, Phys. Rev. Lett. 118, 077001 (2017).

[14] H. Q. Yuan, D. F. Agterberg, N. Hayashi, P. Badica, D. Vandervelde, K. Togano, M. Sigrist, and M. B. Salamon, Phys. Rev. Lett. 97, 017006 (2006).

[15] K. Togano, P. Badica, Y. Nakamori, S. Orimo, H. Takeya, and K. Hirata, Phys. Rev. Lett. 93, 247004 (2004).

[16] A. B. Karki, Y. M. Xiong, I. Vekhter, D. Browne, P. W. Adams, D. P. Young, K. R. Thomas, J. Y. Chan, H. Kim, and R. Prozorov, Phys. Rev. B 82, 064512 (2010).

[17] E. M. Carnicom, W. W. Xie, T. Klimczuk, J. J. Lin, K. Grnicka, Z. Sobczak, N. P. Ong, and R. J. Cava, Sci. Adv. 4, 7969 (2018).

[18] J. M. Lu, O. Zeliuk, I. Leermakers, N. F. Q. Yuan, U. Zeitler, K. T. Law, and J. T. Ye, Science 350, 1353 (2015).

[19] Y. Saito et al., Nat. Phys. 12, 144 (2016).

[20] X. Xi et al., Nat. Phys. 12, 139 (2016).

[21] Y. P. Qi et al., Nat. Commun. 7, 11038 (2016).

[22] E. Sajadi et al., Science 362, 922 (2018).

[23] V. Fatemi et al., Science 362, 926 (2018).

[24] J. Cui et al., Nat. Commun. 10, 2044 (2019).

[25] G. Chang et al., Nat. Mater. 17, 978 (2018).
[26] N. F. Q. Yuan, K. F. Mak, and K. T. Law, Phys. Rev. Lett. 113, 097001 (2014).

[27] B. T. Zhou, N. F. Q. Yuan, H.-L. Jiang, and K. T. Law, Phys. Rev. B 93, 180501(R) (2016).

[28] W.-Y. He et al., Commun. Phys. 1, 40 (2018).

[29] By definition, the gyrotropic point groups are point groups which allow nonzero elements in the rank-two pseudotensors. The gyrotropic point groups are the union of the chiral (enantiomorphic) point groups and the polar point groups (point groups with a polar axis) in addition to $S_{4}$ and $D_{2 d}$ point groups. Please see C. Malgrange, C. Ricolleau, and M. Schlenker, Symmetry and Physical Properties of Crystals (Springer, Heidelberg, 2014).

[30] F. de Juan, A. G. Grushin, T. Morimoto, and J. E. Moore, Nat. Commun. 8, 15995 (2017).

[31] A. A. Abrikosov, L. P. Gorkov, and I. E. Dzyaloshinskii, Methods of Quantum Field Theory in Statistical Physics (PrenticeHall, Englewood Cliffs, NJ, 1963).

[32] See Supplemental Material at http://link.aps.org/supplemental/ 10.1103/PhysRevResearch.2.012073 for the detailed derivation of magnetoelectric susceptibility, the generalization to the multi-band superconductors, the detailed symmetry analysis of magnetoelectric pseudotensor, and the table of candidate superconducting materials.

[33] M. Tinkham, Introduction to Superconductivity (Dover, New York, 2004).

[34] K. V. Samokhin, Ann. Phys. 324, 2385 (2009).

[35] M. Smidman, M. B. Salamon, H. Q. Yuan, and D. F. Agterberg, Rep. Prog. Phys. 80, 036501 (2017).

[36] A. Johansson, J. Henk, and I. Mertig, Phys. Rev. B 93, 195440 (2016).

[37] A. Johansson, J. Henk, and I. Mertig, Phys. Rev. B 97, 085417 (2018).

[38] J. C. R. Sanchez, L. Vila, G. Desfonds, S. Gambarelli, J. P. Attane, J. M. D. Teresa, C. Magen, and A. Fert, Nat. Commun. 4, 2944 (2013).

[39] J. Linder and J. W. A. Robinson, Nat. Phys. 11, 307 (2015).

[40] I. M. Miron et al., Nat. Mater. 9, 230 (2010).

[41] J. Lee, Z. Wang, H. Xie, K. F. Mak, and J. Shan, Nat. Mater. 16, 887 (2017). 